Reprod. Nutr. Dévelop., 1987, 27 (1 B), 269-270.

\title{
Influence de la complémentation de l'herbe de pâturage par de l'orge et de la pulpe de betterave sur la digestion et la synthèse microbienne dans le rumen
}

\author{
A. THÉWIS, D. LEMAL, F. RODRIGUEZ, E. FRANÇOIS $\left({ }^{*}\right)$, Nicole BARTIAUX-THILL $\left({ }^{*}\right)$, \\ Evelyne BAUDART, G. DARDENNE \\ Faculté des Sciences Agronomiques de l'Etat, \\ Passage des Déportés, 2, 5800 Gembloux, Belgique. \\ (*) Centre de Recherches Agronomiques de l'Etat, \\ Avenue de la Faculté d'Agronomie, 22, 5800 Gembloux, Belgique.
}

Summary. The supplementation of fresh grass with barley or sugar beet pulp was studied in sheep. Barley decreased rumen microbial yield while sugar beet pulp or mixture of barley and pulp increased it. Very high values were found for nitrogen degraded in the stomachs.

L'exploitation intensive des pâturages s'accompagne généralement dans nos régions d'une complémentation de l'herbe par un concentré énergétique. L'objectif de ce travail est d'étudier l'influence d'une supplémentation d'orge ou/et de pulpe de betterave sur la digestion de l'herbe de pâturage permanent et plus particulièrement sur la protéosynthèse microbienne dans le rumen du mouton.

Matériel et méthodes. Les béliers utilisés au cours des expériences sont porteurs d'une canule du rumen et d'une canule simple au duodénum. Ils sont maintenus en cage à métabolisme et reçoivent une ration distribuée à raison de $50 \mathrm{~g}$ de $\mathrm{MS} / \mathrm{kg} \mathrm{P}^{0,75}$ en 4 repas égaux à $6 \mathrm{~h}, 12 \mathrm{~h}, 18 \mathrm{~h}$ et $24 \mathrm{~h}$. Elle est composée d'herbe fauchée chaque jour et complémentée pour certains lots d'animaux, par un aliment énergétique représentant $35 \%$ de la MS. A chaque repas, les béliers reçoivent une dose identique de papier imprégné de $\mathrm{Cr}_{2} \mathrm{O}_{3}$. Après une période d'adaptation aux régimes de 10 jours au moins, on mesure la digestibilité globale des rations (7 jours). On prélève ensuite pendant 2 jours, au moment des repas, des échantillons de chyme duodénal. Enfin, pendant les 2 jours suivants, des prélèvements de jus de rumen sont effectués à $11 \mathrm{~h}, 13 \mathrm{~h}$ et $14 \mathrm{~h}$. Les flux de chyme au duodénum sont déterminés à l'aide du $\mathrm{Cr}_{2} \mathrm{O}_{3}$. La part d'azote bactérien dans le flux d'azote non ammoniacal au duodénum est estimée à partir du DAPA dosé au moyen d'un analyseur d'acides aminés du type LKB 4.400. Expérience 1: Neuf béliers sont répartis en 3 lots homogènes et reçoivent les régimes suivants : herbe seule $\left(H_{1}\right)$, herbe plus orge floconnée $\left(H_{1} O\right)$ et herbe plus pulpe de betterave déshydratée $\left(H_{1} P\right)$. L'herbe provient d'une prairie permanente à dominance de ray-grass anglais exploitée au stade montaison $(17,6 \%$ de MAT et $23,7 \%$ de $\mathrm{CB}$ ). Expérience 2. Six béliers issus de l'expérience 1, répartis en 2 lots, reçoivent une ration composée d'herbe seule $\left(\mathrm{H}_{2}\right)$ ou d'herbe plus un mélange en parties égales d'orge floconnée et de pulpe $\left(\mathrm{H}_{2} \mathrm{OP}\right)$. L'herbe provient de la même parcelle que celle de l'expérience 1 mais elle est exploitée au stade pleine épiaison $(11 \%$ de MAT et $28,2 \%$ de $C B$ ).

Travaux subsidiés par le Fonds de la Recherche Fondamentale Collective, Convention 2.4542.84. et la Communauté Economique Européenne, contrat TSD.A-189-B(TT). 
Résultats et discussion. Les digestibilités de la matière organique (MO) des herbes utilisées sont très élevées, particulièrement celle de l'herbe 1 (tabl. 1). Si la valeur mesurée pour cette herbe est supérieure aux données généralement observées sur moutons pour des fourrages verts jeunes, elle est toutefois comparable à celle obtenue sur vaches laitières par Vérité et al. (1984) pour un ray-grass de composition chimique analogue et exploité environ à la même époque de l'année. La complémentation des 2 herbes étudiées améliore encore la digestion de la MO sauf, comme l'ont déjà constaté Grenet et Demarquilly (1977), dans le cas de l'orge. La digestion dans le rumen de la MO est augmentée tant par l'orge que par la pulpe dans le cas de l'herbe 1 ; les différences ne sont toutefois pas significatives.

La part de l'azote alimentaire dégradée dans le rumen est très importante (tabl. 1). Bien que l'utilisation du DAPA comme marqueur de l'azote bactérien reste criticable, les valeurs obtenues pour l'herbe seule concordent avec celles mentionnées par Demarquilly et Jarrige (1982) pour les fourrages verts. Par ailleurs, les valeurs observées pour les rations mixtes à base de pulpes paraissent anormalement élevées (fermentescibilité in sacco de la pulpe <60).

TABL. 1. - Influence de la supplémentation énergétique de 2 herbes de prairie permanente sur la digestion de la matière organique (MO) et des matières azotées totales (MAT) chez le mouton, estimation de la protéosynthèse microbienne $(\bar{x} \pm \sigma)$.

\begin{tabular}{|c|c|c|c|c|c|}
\hline Régimes & $H_{1}$ & $\mathrm{H}_{1} \mathrm{O}$ & $\mathrm{H}_{1} \mathrm{P}$ & $\mathrm{H}_{2}$ & $\mathrm{H}_{2} \mathrm{OP}$ \\
\hline Nombre d'animaux & 3 & 2 & 3 & 2 & 3 \\
\hline MO ingérée $(g / j)$ & $966 \pm 41$ & $989 \pm 92$ & $974 \pm 48$ & $1000 \pm 23$ & $1066 \pm 83$ \\
\hline Digestibilité MO (\%) & 80,3 & 79,5 & 83,0 & $75,2 \pm 0,1$ & $77,7 \pm 1,1$ \\
\hline Digestibilité $\mathrm{MO}$ dans rumen $(\%)$ & $55,2 \pm 2,7$ & $57,6 \pm 3,3$ & $58,5 \pm 2,8$ & $55,1 \pm 1,6$ & $54,2 \pm 1,2$ \\
\hline Azote ingéré $(g / j)$ & $31,8 \pm 1,4$ & $27,3 \pm 2,5$ & $27,8 \pm 1,4$ & $18,8 \pm 0,5$ & $19,7 \pm 0,8$ \\
\hline Digestibilité MAT (\%) & $80,5 \pm 1,0$ & $75,8 \pm 0,2$ & $77,2 \pm 0,5$ & $71,1 \pm 0,5$ & $71,0 \pm 1,0$ \\
\hline $\mathrm{N}-\mathrm{NH}_{3}$ rumen $(\mathrm{mg} / \mathrm{l})$ & $242 \pm 51$ & $220 \pm 39$ & $179 \pm 29$ & $133 \pm 32$ & $121 \pm 17$ \\
\hline Flux duodénal $\mathrm{N}$ non $\mathrm{NH}_{3}(\mathrm{~g} / \mathrm{j})$ & $23,8 \pm 1,6$ & $21,6 \pm 2,9$ & $26,8 \pm 3,3$ & $20,0 \pm 1,2$ & $24,7 \pm 3,1$ \\
\hline $\begin{array}{l}\text { Flux duodénal } N \text { bactérien }(\mathrm{g} / \mathrm{j}) \\
\mathrm{N} \text { alimentaire dégradé }\end{array}$ & $16,2 \pm 1,5$ & $15,1 \pm 3,4$ & $20,1 \pm 1,1$ & $16,7 \pm 1,0$ & $20,8 \pm 2,5$ \\
\hline (\% N ingéré) $\left({ }^{*}\right)$ & $84,4 \pm 3,9$ & $85,2 \pm 2,2$ & $85,6 \pm 6,5$ & $90,4 \pm 3,7$ & $91,1 \pm 6,2$ \\
\hline $\mathrm{N}$ bactérien $/ \mathrm{kg} \operatorname{MODR}\left({ }^{* *}\right)(\mathrm{g} / \mathrm{kg})$ & $30,7 \pm 4,7$ & $24,1 \pm 1,4$ & $35,5 \pm 2,7$ & $30,2 \pm 1,4$ & $34,1 \pm 2,1$ \\
\hline
\end{tabular}

(*) Calculé en supposant $N$ endogène : $2 \mathrm{~g} /$ jour ; $N$ protozoaire : $2 \%$ de $N$ ingéré ; $\left({ }^{*}\right)$ MODR matière organique digérée dans le rumen.

Les rendements de la protéosynthèse microbienne font ressortir la supériorité de la pulpe de betterave par rapport à l'orge comme substrat énergétique pour les bactéries du rumen en cas de supplémentation d'herbes très digestibles renfermant une part importante d'azote et de glucides solubles. La dégradation progressive mais élevée des constituants pariétaux de la pulpe permet une utilisation plus rationnelle de l'ammoniac dégagé dans le rumen que la ferrientation rapide de l'amidon de l'orge qui s'ajoute encore à celles des glucides solubles du fourrage.

Grenet E., Demarquilly C., 1977. Ann. Zootech., 26, 481-501.

Demarquilly C., Jarrige R., 1982. In: Forage protein conservation and utilisation. Commission of European Communities, Dublin, 147-172.

Vérité R., Rémond B., Journet M., 1984. Can. J. anim. Sci., 64 (Suppl.), 328-329. 